\title{
Revealing the molecular signaling pathways of mucus stasis in cystic fibrosis
}

\author{
Susan E. Birket and Steven M. Rowe \\ Gregory Fleming James Cystic Fibrosis Research Center, University of Alabama at Birmingham, Birmingham, Alabama, USA.
}

\begin{abstract}
Mucus obstruction is a hallmark of cystic fibrosis (CF) airway disease, leading to chronic infection, dysregulated inflammation, and progressive lung disease. As mucus hyperexpression is a key component in the initiation and perpetuation of airway obstruction, the triggers underlying mucin release must be identified and understood. In this issue of the JCI, Chen et al. sought to delineate the mechanisms that allow IL-1 $\alpha / \mathrm{IL}-1 \beta$ to perpetuate the mucoinflammatory environment characteristic of the CF airway. The authors demonstrated that IL- $1 \alpha$ and IL-1 $\beta$ stimulated non-CF human bronchial epithelial (HBE) cells to upregulate and secrete both MUC5B and MUC5AC in a dose-dependent manner, an effect that was neutralized by the inhibition of the IL-1 $\alpha / I L-1 \beta$ receptor (IL-1R1). Further experiments using mouse models and excised lung tissue identified contributors that drive a vicious feedback cycle of hyperconcentrated mucus secretions and persistent inflammation in the CF airway, factors that are likely at the nidus of progressive lung disease.
\end{abstract}

\section{The mucoinflammatory} cascade in cystic fibrosis

While complete airway occlusion and mucus plugging are most commonly associated with late-stage cystic fibrosis (CF) disease, evidence collected over the last decade has demonstrated that these events begin at a young age. Neonatal $\mathrm{CFTR}^{-/}$piglets show evidence of air trapping prior to bacterial exposures (1), corresponding to similar findings detected in patients with $\mathrm{CF}$ in the first few years of life (2). More recent data suggests that the mucoobstructive phenotype that results in bronchiectasis and pulmonary function decline is present and damaging even in the absence of overt infection (3). Patients at the earliest stages of the disease have evidence of neutrophil influx, neutrophil elastase, and increased mucin concentrations (4). In a ferret model of CF, these events occur despite aggressive bacterial eradication (5), suggesting that infection control alone will not prevent progression of lung disease. Cell culture, animal tissue, and human studies have shown that airway dehydration, a contributor to mucus hyperconcentration, is an important factor in mucus stasis, compounding the effects of elevated mucus viscosity that may be inherent to CF mucus (6-8). Taking these data together, it is evident that the mucoinflammatory cascade is sufficient to cause structural airway damage and therefore an attractive potential target for CF therapy.

IL-1, a family of cytokines predominant in the proinflammatory immune response, has previously been associated with both airway inflammation (9) and mucus obstruction (10) in CF; however, the specific pathways that underlie increased mucin secretion were poorly understood. In this issue, Chen and colleagues used complementary human cell and animal models to identify the respective roles of IL-1R1, SPDEF, and ERN2 in the regula-

Related Article: p. 4433

Conflict of interest: The authors have declared that no conflict of interest exists.

Copyright: () 2019, American Society for Clinical Investigation.

Reference information: / Clin Invest. 2019;129(10):4089-4090. https://doi.org/10.1172/JCI131652.

tion of mucins MUC5B and MUC5AC, to reveal molecular pathways with potential treatment opportunities (11).

Previously, the supernatants of airway mucopurulent secretions (SAMS) from people with $C F$ were used to induce mucin secretion in human bronchial epithelial (HBE) cells (12). Chen et al. targeted IL-1 $\beta$ as a major driver of mucin secretion because it is highly expressed in SAMS (12) and showed that treatment of non-CF HBE cells with IL-1 $\beta$ stimulates MUC5B and MUC5AC upregulation. Likewise, $\mathrm{CF}$ HBE cells overexpressed mucin, an effect predominated by MUC5B. Interestingly, IL-1 $\beta$, but not IL-13 (a TH2 cytokine prevalent in asthma that predominantly increases MUC5AC secretion), caused an increase in the solid content of the apical secretions only from CF $\mathrm{HBE}$ cells. As a compliment of previous reports that apical secretions from CF HBE contain increased solid content (12), Chen et al. showed that the solid component is dominated by elevated MUC5B. In normal mice, IL-1 $\beta$ treatment as well as SAMS administration to the airways reproduced mucin upregulation. Conversely, mucin secretion was abrogated in murine models lacking either IL-1R1 or SPDEF, a key mediator in $M u c 5 b$ expression (13). These results indicate that IL-1 $\beta$ increases $M u c 5 b$ via SPDEF signaling. Chen and colleagues went on to show that ERN2 expression decreased in Spdef/- mice treated with IL-1 $\beta$, suggesting that SPDEF in turn regulates ERN2. Finally, MUC5B, $I L 1 B, S P D E F$, and ERN2 mRNA expression were confirmed in lung explant tissue derived from individual patients with $\mathrm{CF}$, and each marker was detected throughout the airway into the distal regions of the lung; however, these markers were largely absent in non-CF lung tissue.

These studies provide key pathways that serve to initiate and perpetuate mucus obstruction. While the complementary nature of the findings in the models used, ranging from airway cell culture mono- 
layers to genetically altered mice, clearly invoke pathways relevant to obstruction at the airway surface, a crucial aspect of $\mathrm{CF}$ pathogenesis and particularly relevant to the small airways, it should also be noted that these systems lack widespread submucosal glands, which contribute a significant quantity of MUC5B in larger airways. Submucosal glands are an important facet of human lung disease that may operate under distinct mechanisms (13-15). Determining whether these pathways also drive gland obstruction events and their relative contribution to lung pathology in the small or large diameter airways should be a high priority for $\mathrm{CF}$ research.

These studies also have implications beyond CF lung disease and could yield insights into additional obstructive airway diseases plagued by mucus occlusion. Future experiments to identify inconsistencies in the effects of IL-13 administration on both MUC5B and MUC5AC from cell to animal models may reveal other mucus obstruction events in asthma. Given commonalities in mucus-overexpression phenotypes and their underlying contributors, chronic bronchitis and other forms of smoking related lung disease are a high priority $(16,17)$. Whether elevated MUC5B expression associated with idiopathic pulmonary fibrosis is related to mucus stasis also deserves exploring $(18,19)$.

\section{Preventing mucus accumulation}

The study by Chen et al. has clear therapeutic implications, demonstrating that genetic knockdown of IL-1R1 abrogated mucus hyperconcentration, even in the presence of the stimulatory effect of SAMS or IL-1 $\beta$. Further research is warranted to determine whether receptor blockade by small molecules or expression inhibitors will prove similarly efficacious in preventing mucus accumulation. These data suggest that, while the upstream targets, SPDEF or ERN2, may serve as alternative therapeutic approaches, they could also have broad effects on the lung that will require careful evaluation. Determining whether intervention through this signaling pathway alters mucus viscosity, mucociliary transport rates, or mucus stasis events in CF airways is a clear future direc- tion. Certainly antiinflammatory targeting has had mixed success in CF therapeutics, due likely to overlapping mechanisms (20), a theme seen also in other lung diseases. We would expect targeting the IL-1 $\beta$ signaling pathway in the more complex and infected environment of the CF lung to present similar treatment challenges. Nevertheless, even partially reducing mucus burden within the CF lung is likely to have therapeutic value and could act in concert with other mucolytic therapies.

As summarized at the ATS President's Symposium this year, a decade ago, the lung community began to transition from an emphasis on physiology to one on the molecular pathways (21). Data presented by Chen and colleagues identify a signaling pathway that underlies the plaguing problem of mucus obstruction, a disease phenotype that is present in many airway diseases. As the pulmonary field continues to link molecular discoveries to the next era of physiologic assessments of the lung, there is potential for providing impactful mechanistic insights that increase the likelihood of developing successful therapies.

\section{Acknowledgments}

The authors acknowledge support from the NIH (P30DK072482, R35HL135816, K08HL131867) and the Cystic Fibrosis Foundation (ROWE19R0) as well as many helpful conversations within the Mucus Clearance Consortium and Mucociliary Interaction Gordon Conference series.

Address correspondence to: Steven M. Rowe, MCLM 706, Birmingham, Alabama 35294-0005, USA. Phone: 205.934.9640; Email: smrowe@uab.edu.

1. Adam RJ, et al. Air trapping and airflow obstruction in newborn cystic fibrosis piglets. Am J Respir Crit Care Med. 2013;188(12):1434-1441.

2. Stick SM, et al. Bronchiectasis in infants and preschool children diagnosed with cystic fibrosis after newborn screening. J Pediatr. 2009;155(5):623-628.e1.

3. Rosenow T, et al. The cumulative effect of inflammation and infection on structural lung disease in early cystic fibrosis. Eur Respir J. 2019;54(1):1801771.

4. Esther CR, et al. Mucus accumulation in the lungs precedes structural changes and infection in children with cystic fibrosis. Sci Transl Med. 2019;11(486):eaav3488.
5. Rosen BH, et al. Infection is not required for mucoinflammatory lung disease in CFTR-knockout ferrets. Am J Respir Crit Care Med. 2018;197(10):1308-1318.

6. Birket SE, et al. Combination therapy with cystic fibrosis transmembrane conductance regulator modulators augment the airway functional microanatomy. Am J Physiol Lung Cell Mol Physiol. 2016;310(10):L928-L939.

7. Birket SE, et al. A functional anatomic defect of the cystic fibrosis airway. Am J Respir Crit Care Med. 2014;190(4):421-432.

8. Leung HM, et al. Intranasal micro-optical coherence tomography imaging for cystic fibrosis studies. Sci Transl Med. 2019;11(504):eaav3505.

9. Montgomery ST, et al. Interleukin-1 is associated with inflammation and structural lung disease in young children with cystic fibrosis. J Cyst Fibros. 2018;17(6):715-722.

10. Fritzsching B, et al. Hypoxic epithelial necrosis triggers neutrophilic inflammation via IL-1 receptor signaling in cystic fibrosis lung disease. Am J Respir Crit Care Med. 2015;191(8):902-913.

11. Chen $\mathrm{G}$, et al. IL-1 $\beta$ dominates the promucin secretory cytokine profile in cystic fibrosis. J Clin Invest. 2019;129(10):4433-4450.

12. Abdullah LH, et al. Mucin production and hydration responses to mucopurulent materials in normal versus cystic fibrosis airway epithelia. Am J Respir Crit Care Med. 2018;197(4):481-491.

13. Ermund A, Meiss LN, Dolan B, Bähr A, Klymiuk $\mathrm{N}$, Hansson GC. The mucus bundles responsible for airway cleaning are retained in cystic fibrosis and by cholinergic stimulation. Eur Respir J. 2018;52(2):1800457.

14. Cho HJ, Joo NS, Wine JJ. Defective fluid secretion from submucosal glands of nasal turbinates from CFTR-/- and CFTR ( $\triangle$ F508/ $\triangle \mathrm{F} 508)$ pigs. PLoS One. 2011;6(8):e24424.

15. Salinas D, et al. Submucosal gland dysfunction as a primary defect in cystic fibrosis. FASEB J. 2005;19(3):431-433.

16. Fahy JV, Dickey BF. Airway mucus function and dysfunction. NEnglJMed.2010;363(23):2233-2247.

17. Raju SV, Solomon GM, Dransfield MT, Rowe SM. Acquired cystic fibrosis transmembrane conductance regulator dysfunction in chronic bronchitis and other diseases of mucus clearance. Clin Chest Med. 2016;37(1):147-158.

18. Hunninghake GM, et al. MUC5B promoter polymorphism and interstitial lung abnormalities. N Engl J Med. 2013;368(23):2192-2200.

19. Hancock LA, et al. Muc5b overexpression causes mucociliary dysfunction and enhances lung fibrosis in mice. Nat Commun. 2018;9(1):5363.

20. Cantin AM, Hartl D, Konstan MW, Chmiel JF. Inflammation in cystic fibrosis lung disease: Pathogenesis and therapy. J Cyst Fibros. 2015;14(4):419-430.

21. Gibbons G, Drazen J, Whitsett J, Han M, Calfee C. 50 Years of Pulmonary Science. President's symposium presented at: American Thoracic Society International Conference; May 17-22, 2019; Dallas, Texas, USA. 